\title{
Invasion traveling wave solutions of a competitive system with dispersal
}

Shuxia Pan ${ }^{1 *}$ and Guo Lin²

*Correspondence: shxpan@yeah.net 'Department of Applied Mathematics, Lanzhou University of Technology, Lanzhou, Gansu 730050, People's Republic of China Full list of author information is

available at the end of the article

\begin{abstract}
This paper is concerned with the invasion traveling wave solutions of a Lotka-Volterra type competition system with nonlocal dispersal, the purpose of which is to formulate the dynamics between the resident and the invader. By constructing upper and lower solutions and passing to a limit function, the existence of traveling wave solutions is obtained if the wave speed is not less than a threshold. When the wave speed is smaller than the threshold, the nonexistence of invasion traveling wave solutions is proved by the theory of asymptotic spreading.
\end{abstract}

MSC: $35 \mathrm{CO} 7 ; 35 \mathrm{~K} 57 ; 37 \mathrm{C} 65$

Keywords: comparison principle; asymptotic spreading; upper and lower solutions; invasion waves

\section{Introduction}

In the past decades, much attention has been paid to the spatial propagation modes of the following Lotka-Volterra type diffusion system:

$$
\left\{\begin{array}{l}
\frac{\partial u_{1}(x, t)}{\partial t}=d_{1} \Delta u_{1}(x, t)+r_{1} u_{1}(x, t)\left[1-u_{1}(x, t)-b_{1} u_{2}(x, t)\right] \\
\frac{\partial u_{2}(x, t)}{\partial t}=d_{2} \Delta u_{2}(x, t)+r_{2} u_{2}(x, t)\left[1-u_{2}(x, t)-b_{2} u_{1}(x, t)\right]
\end{array}\right.
$$

in which all the parameters are positive and $x \in \mathbb{R}, t>0, u_{1}, u_{2}$ are two competitors. Many investigators considered its traveling wave solutions connecting different spatial homogeneous steady states such as the existence, monotonicity, minimal wave speed and stability; see [1-16].

In particular, if $b_{1}<1<b_{2}$ holds in (1.1), then the corresponding reaction system has a stable equilibrium $(1,0)$ and an unstable one $(0,1)$. With the condition $b_{1}<1<b_{2}$, many papers including $[2,3,5,6,8,16]$ studied the traveling wave solutions connecting $(1,0)$ with $(0,1)$. These traveling wave solutions can formulate the spatial exclusive process between the resident $u_{2}$ and the invader $u_{1}$ so that the minimal wave speed reflecting the invasion speed of the invader becomes a hot topic in these works; we refer to Shigesada and Kawasaki [17] for some examples of the corresponding biological records and the literature importance of invasion speed. Moreover, the similar problem was also discussed in different spatial media such as the lattice differential systems in Guo and Liang [4], Guo and $\mathrm{Wu}[18]$.

o 2012 Pan and Lin: licensee Springer. This is an Open Access article distributed under the terms of the Creative Commons Attribution License (http://creativecommons.org/licenses/by/2.0), which permits unrestricted use, distribution, and reproduction in any medium, provided the original work is properly cited. 
In this paper, we consider the minimal wave speed of traveling wave solutions in the following nonlocal dispersal system (see Yu and Yuan [19]):

$$
\left\{\begin{aligned}
\frac{\partial u_{1}(x, t)}{\partial t}= & d_{1}\left[\int_{\mathbb{R}} J_{1}(x-y) u_{1}(y, t) d y-u_{1}(x, t)\right] \\
& +r_{1} u_{1}(x, t)\left[1-u_{1}(x, t)-b_{1} u_{2}(x, t)\right], \\
\frac{\partial u_{2}(x, t)}{\partial t}= & d_{2}\left[\int_{\mathbb{R}} J_{2}(x-y) u_{2}(y, t) d y-u_{2}(x, t)\right] \\
& +r_{2} u_{2}(x, t)\left[1-u_{2}(x, t)-b_{2} u_{1}(x, t)\right],
\end{aligned}\right.
$$

in which $x \in \mathbb{R}, t>0, u_{1}(x, t)$ and $u_{2}(x, t)$ denote the densities of two competitors at time $t$ and location $x \in \mathbb{R}$, all the parameters are positive and

$$
b_{1}<1<b_{2} \text {, }
$$

$J_{i}: \mathbb{R} \rightarrow \mathbb{R}, i=1,2$, are probability functions formulating the random dispersal of individuals and satisfy the following assumptions:

(J1) $J_{i}$ is nonnegative and Lebesgue measurable for each $i=1,2$;

(J2) for any $\lambda \in \mathbb{R}, \int_{\mathbb{R}} J_{i}(y) e^{\lambda y} d y<\infty, i=1,2$;

(J3) $\int_{\mathbb{R}} J_{i}(y) d y=1, J_{i}(y)=J_{i}(-y), y \in \mathbb{R}, i=1,2$.

In (1.2), the spatial migration of individuals is formulated by the so-called dispersal operator, which has significant sense in population dynamics. For example, in the patch models of population dynamics [20], the rate of immigration into a patch from a particular other patch is usually taken as proportional to the local population, and the dispersal can be regarded as the extension of these ideas to a continuous media model. Such a diffusion mechanism also arises from physics processes with long range effect and other disciplines [13], and the dynamics of evolutionary systems with dispersal effect has been widely studied in recent years; we refer to [13, 21-32] and the references cited therein.

Hereafter, a traveling wave solution of (1.2) is a special solution of the form

$$
u_{1}(x, t)=\phi_{1}(\xi), \quad u_{2}(x, t)=\phi_{2}(\xi), \quad \xi=x+c t,
$$

where $c>0$ is the wave speed at which the wave profile $\left(\phi_{1}, \phi_{2}\right) \in C^{1}\left(\mathbb{R}, \mathbb{R}^{2}\right)$ propagates in spatial media $\mathbb{R}$. Thus, $\left(\phi_{1}, \phi_{2}\right)$ with $c>0$ must satisfy

$$
\left\{\begin{aligned}
c \phi_{1}^{\prime}(\xi)= & d_{1}\left[\int_{\mathbb{R}} J_{1}(\xi-y) \phi_{1}(y) d y-\phi_{1}(\xi)\right] \\
& +r_{1} \phi_{1}(\xi)\left[1-\phi_{1}(\xi)-b_{1} \phi_{2}(\xi)\right], \quad \xi \in \mathbb{R}, \\
c \phi_{2}^{\prime}(\xi)= & d_{2}\left[\int_{\mathbb{R}} J_{2}(\xi-y) \phi_{2}(y) d y-\phi_{2}(\xi)\right] \\
& +r_{2} \phi_{2}(\xi)\left[1-\phi_{2}(\xi)-b_{2} \phi_{1}(\xi)\right], \quad \xi \in \mathbb{R} .
\end{aligned}\right.
$$

Moreover, we also require the following asymptotic boundary conditions:

$$
\lim _{\xi \rightarrow-\infty}\left(\phi_{1}(\xi), \phi_{2}(\xi)\right)=(0,1), \quad \lim _{\xi \rightarrow \infty}\left(\phi_{1}(\xi), \phi_{2}(\xi)\right)=(1,0)
$$

From the viewpoint of ecology, a traveling wave solution satisfying (1.4)-(1.5) can model the population invasion process: at any fixed $x \in \mathbb{R}$, only $u_{2}$ (the resident) can be found 
long time ago $(t \rightarrow-\infty$ such that $x+c t \rightarrow-\infty)$, but after a long time $(t \rightarrow \infty$ such that $x+c t \rightarrow \infty$ ), only $u_{1}$ (the invader) can be seen. Therefore, we call a traveling wave solution satisfying (1.4)-(1.5) an invasion traveling wave solution.

To obtain the existence of (1.4)-(1.5) if the wave speed is larger than a threshold depending on $J_{1}, d_{1}, r_{1}$ and $b_{1}$, we construct proper upper and lower solutions and use the results in Pan et al. [33]. If the wave speed is the threshold, the existence of traveling wave solutions is proved by passing to a limit function. Finally, when the wave speed is smaller than the threshold, the nonexistence of traveling wave solutions is established by the theory of asymptotic spreading developed by Jin and Zhao [34]. For more results on the traveling wave solutions of evolutionary systems with nonlocal dispersal, we refer to Bates et al. [22], Coville and Dupaigne [35, 36], Li et al. [37], Lv [38], Pan [39], Pan et al. [33, 40], Sun et al. [41], Wu and Liu [42], Xu and Weng [43], Zhang et al. [44]. In particular, when $b_{1}, b_{2} \in(0,1)$ hold in (1.2), Yu and Yuan [19] established the existence of traveling wave solutions connecting $(0,0)$ with

$$
\left(\frac{1-b_{2}}{1-b_{1} b_{2}}, \frac{1-b_{1}}{1-b_{1} b_{2}}\right)
$$

In addition, Li and Lin [45] and Zhang et al. [46] investigated the existence of positive traveling wave solutions of (1.2) for $b_{1}<0, b_{2}<0$ and $b_{1} b_{2}<0$, respectively.

The rest of this paper is organized as follows. In Section 2, we give some preliminaries. By constructing upper and lower solutions and using a limit process, the existence of traveling wave solutions is established in Section 3. In the last section, we obtain the nonexistence of traveling wave solutions.

\section{Preliminaries}

In this paper, we shall use the standard partial order in $\mathbb{R}^{2}$. Moreover, denote

$$
X=\left\{\mathbf{u}: \mathbf{u} \text { is a bounded and uniformly continuous function from } \mathbb{R} \text { to } \mathbb{R}^{2}\right\},
$$

then $X$ is a Banach space equipped with the standard supremum norm. If $\mathbf{a}, \mathbf{b} \in \mathbb{R}^{2}$ with $\mathbf{a} \leq \mathbf{b}$, then

$$
X_{[\mathbf{a}, \mathbf{b}]}=\{\mathbf{u} \in X: \mathbf{a} \leq \mathbf{u}(\xi) \leq \mathbf{b}, \xi \in \mathbb{R}\} .
$$

In order to apply the comparison principle, we first make a change of variables to obtain a cooperative system. Let $\phi_{1}^{*}=\phi_{1}, \phi_{2}^{*}=1-\phi_{2}$, and drop the star for the sake of convenience, then (1.4) becomes

$$
\left\{\begin{array}{l}
c \phi_{1}^{\prime}(\xi)=d_{1}\left[\int_{\mathbb{R}} J_{1}(\xi-y) \phi_{1}(y) d y-\phi_{1}(\xi)\right]+r_{1} \phi_{1}(\xi)\left[1-b_{1}-\phi_{1}(\xi)+b_{1} \phi_{2}(\xi)\right] \\
c \phi_{2}^{\prime}(\xi)=d_{2}\left[\int_{\mathbb{R}} J_{2}(\xi-y) \phi_{2}(y) d y-\phi_{2}(\xi)\right]+r_{2}\left[1-\phi_{2}(\xi)\right]\left[b_{2} \phi_{1}(\xi)-\phi_{2}(\xi)\right] .
\end{array}\right.
$$

At the same time, (1.5) will be

$$
\lim _{\xi \rightarrow-\infty}\left(\phi_{1}(\xi), \phi_{2}(\xi)\right)=(0,0), \quad \lim _{\xi \rightarrow \infty}\left(\phi_{1}(\xi), \phi_{2}(\xi)\right)=(1,1)
$$


Take $\beta=2\left(d_{1}+d_{2}+r_{1}+r_{2}+1\right)\left(1+b_{1}+b_{2}\right)$ and

$$
\begin{aligned}
& H_{1}\left(\phi_{1}, \phi_{2}\right)(\xi) \\
& \quad=d_{1} \int_{\mathbb{R}} J_{1}(\xi-y) \phi_{1}(y) d y+\left(\beta-d_{1}\right) \phi_{1}(\xi)+r_{1} \phi_{1}(\xi)\left[1-b_{1}-\phi_{1}(\xi)+b_{1} \phi_{2}(\xi)\right], \\
& H_{2}\left(\phi_{1}, \phi_{2}\right)(\xi) \\
& \quad=d_{2} \int_{\mathbb{R}} J_{2}(\xi-y) \phi_{2}(y) d y+\left(\beta-d_{2}\right) \phi_{2}(\xi)+r_{2}\left[1-\phi_{2}(\xi)\right]\left[b_{2} \phi_{1}(\xi)-\phi_{2}(\xi)\right],
\end{aligned}
$$

then $H_{i}$ is monotone in the functional sense if $\left(\phi_{1}, \phi_{2}\right) \in X_{[\mathbf{0}, \mathbf{1}]}$. Applying these notations, we further define an operator $F=\left(F_{1}, F_{2}\right): X_{[\mathbf{0 , 1}]} \rightarrow X_{[\mathbf{0 , 1}]}$ as follows:

$$
F_{i}\left(\phi_{1}, \phi_{2}\right)(\xi)=\frac{1}{c} \int_{-\infty}^{\xi} e^{-\frac{\beta}{c}(\xi-s)} H_{i}\left(\phi_{1}, \phi_{2}\right)(s) d s, \quad i=1,2 .
$$

Clearly, a fixed point of $\left(F_{1}, F_{2}\right)$ in $X$ satisfies (2.1), and a solution of (2.1) is also a fixed point of $F$. To continue our discussion, we also introduce the following definition.

Definition 2.1 Assume that $\left(\rho_{1}, \rho_{2}\right) \in X_{[\mathbf{0}, \mathbf{1}]}$. If $\rho_{1}, \rho_{2}$ are differentiable on $\mathbb{R} \backslash \mathbb{T}$, here $\mathbb{T}$ contains finite points, and the derivatives are essentially bounded so that

$$
\left\{\begin{aligned}
c \rho_{1}^{\prime}(\xi) \geq(\leq) & d_{1}\left[\int_{\mathbb{R}} J_{1}(\xi-y) \rho_{1}(y) d y-\rho_{1}(\xi)\right] \\
& +r_{1} \rho_{1}(\xi)\left[1-b_{1}-\rho_{1}(\xi)+b_{1} \rho_{2}(\xi)\right] \\
c \rho_{2}^{\prime}(\xi) \geq(\leq) & d_{2}\left[\int_{\mathbb{R}} J_{2}(\xi-y) \rho_{2}(y) d y-\rho_{2}(\xi)\right] \\
+ & r_{2}\left[1-\rho_{2}(\xi)\right]\left[b_{2} \rho_{1}(\xi)-\rho_{2}(\xi)\right]
\end{aligned}\right.
$$

for $\xi \in \mathbb{R} \backslash \mathbb{T}$, then it is an upper (a lower) solution of (2.1).

Using Pan et al. [33], Theorem 3.2, we obtain the following conclusion.

Lemma 2.2 Assume that $\left(\bar{\phi}_{1}(\xi), \bar{\phi}_{2}(\xi)\right)$ is an upper solution of $(2.1)$, while $\left(\underline{\phi}_{1}(\xi), \underline{\phi}_{2}(\xi)\right)$ is a lower solution of (2.1). Also, suppose that

(P1) $\left(\underline{\phi}_{1}(\xi), \underline{\phi}_{2}(\xi)\right) \leq\left(\bar{\phi}_{1}(\xi), \bar{\phi}_{2}(\xi)\right)$;

(P2) $\lim _{\xi \rightarrow-\infty}\left(\bar{\phi}_{1}(\xi), \bar{\phi}_{2}(\xi)\right)=(0,0), \lim _{\xi \rightarrow \infty}\left(\bar{\phi}_{1}(\xi), \bar{\phi}_{2}(\xi)\right)=(1,1)$;

(P3) $\sup _{s<\xi} \underline{\phi}_{i}(s) \leq \inf _{s>\xi} \bar{\phi}_{i}(s)$ for all $\xi \in \mathbb{R}, i=1,2$, and $\sup _{\xi \in \mathbb{R}} \underline{\phi}_{1}(\xi)>0$.

Then (2.1)-(2.2) has a positive monotone solution $\left(\phi_{1}(\xi), \phi_{2}(\xi)\right)$ such that

$$
\left(\underline{\phi}_{1}(\xi), \underline{\phi}_{2}(\xi)\right) \leq\left(\phi_{1}(\xi), \phi_{2}(\xi)\right) \leq\left(\bar{\phi}_{1}(\xi), \bar{\phi}_{2}(\xi)\right)
$$

We now consider the following initial value problem:

$$
\left\{\begin{array}{l}
\frac{\partial u(x, t)}{\partial t}=d\left[\int_{\mathbb{R}} J(x-y) u(y, t) d y-u(x, t)\right]+r u(x, t)[1-u(x, t)], \\
u(x, 0)=\phi(x), \quad x \in \mathbb{R},
\end{array}\right.
$$

where $J$ satisfies (J1) to (J3), $d>0$ and $r>0$ are constants, and the initial value $\phi(x) \in$ $C(\mathbb{R}, \mathbb{R})$ with

$$
C(\mathbb{R}, \mathbb{R})=\{\phi: \phi \text { is a bounded and uniformly continuous function from } \mathbb{R} \text { to } \mathbb{R}\} \text {. }
$$


In addition, let $C^{+}$be a subset of $C$ defined by

$$
C^{+}=\{\phi \in C: \phi(x) \geq 0, x \in \mathbb{R}\}
$$

In Jin and Zhao [34], the authors investigated the asymptotic spreading of a periodic population model with spatial dispersal. Note that the parameters in (2.4) are positive constants, then [34], Theorem 2.1, implies the following result.

Lemma 2.3 Assume that $\phi(x) \in C^{+}$. Then (2.4) has a unique solution $u(x, t)$ such that

$$
u(x, t) \geq 0, \quad x \in \mathbb{R}, t>0 .
$$

In particular, if $\phi(x) \in C_{[0, a]}$ with some $a \geq 1$, then

$$
0 \leq u(x, t) \leq a, \quad x \in \mathbb{R}, t>0 .
$$

Furthermore, we can also apply the results of Jin and Zhao [34], Theorem 3.5, since the assumptions (H1) and (H2) of [34] are clear. Define

$$
c_{1}=\inf _{\lambda>0} \frac{d\left[\int_{\mathbb{R}} J(y) e^{\lambda y} d y-1\right]+r}{\lambda} .
$$

Then Jin and Zhao [34], Theorem 3.5, indicates the following conclusion.

Lemma 2.4 Assume that $\phi(x) \in C^{+}$admits nonempty support. Then

$$
\liminf _{t \rightarrow \infty} \inf _{|x|<c t} u(x, t)=\limsup _{t \rightarrow \infty} \sup _{|x|<c t} u(x, t)=1 \quad \text { for any } c<c_{1}
$$

where $u(x, t)$ is defined by (2.4).

\section{Existence of traveling wave solutions}

In this section, we shall prove the existence of positive solutions of (2.1)-(2.2). Let

$$
\Delta_{1}(\lambda, c)=d_{1}\left[\int_{\mathbb{R}} J_{1}(y) e^{\lambda y} d y-1\right]-c \lambda+r_{1}\left(1-b_{1}\right)
$$

for any $\lambda \geq 0, c>0$.

Lemma 3.1 There exists a constant $c^{*}>0$ such that the following items hold.

(1) For each $c>c^{*}, \Delta_{1}(\lambda, c)=0$ has two positive real roots $\lambda_{1}(c)<\lambda_{2}(c)$.

(2) If $c=c^{\prime \prime}$, then there exists $\lambda\left(c^{*}\right)>0$ such that $\Delta_{1}\left(\lambda\left(c^{*}\right), c^{\prime \prime}\right)=0$ and $\Delta_{1}\left(\lambda, c^{*}\right)>0$ for any $\lambda \neq \lambda\left(c^{*}\right)$.

(3) If $c<c^{*}$, then $\Delta_{1}(\lambda, c)>0$ for any $\lambda \geq 0$.

The above result is clear and we omit the proof here. Using these constants, we can prove the following conclusion. 
Theorem 3.2 Assume that $c>c$ and one of the following two items holds.

(1) $b_{1} b_{2}>1$ and

$$
d_{2}\left[\int_{\mathbb{R}} J_{2}(y) e^{\lambda_{1}(c) y} d y-1\right]-c \lambda_{1}(c)+r_{2}\left(b_{1} b_{2}-1\right) \leq 0 .
$$

(2) $b_{1} b_{2} \leq 1$ and

$$
d_{2}\left[\int_{\mathbb{R}} J_{2}(y) e^{\lambda_{1}(c) y} d y-1\right]-c \lambda_{1}(c) \leq 0 .
$$

Then (2.1)-(2.2) has a monotone solution.

Proof Define continuous functions as follows:

$$
\bar{\phi}_{1}(\xi)=\min \left\{e^{\lambda_{1}(c) \xi}, 1\right\}, \quad \bar{\phi}_{2}(\xi)=\min \left\{e^{\lambda_{1}(c) \xi} / b_{1}, 1\right\} .
$$

Claim A: $\left(\bar{\phi}_{1}(\xi), \bar{\phi}_{2}(\xi)\right)$ is an upper solution to (2.1).

Moreover, let $\underline{\phi}_{2}(\xi)=0$ hold and $\underline{\phi}_{1}(\xi)$ satisfy

$$
c \phi_{1}^{\prime}(\xi)=d_{1}\left[\int_{\mathbb{R}} J_{1}(\xi-y) \phi_{1}(y) d y-\phi_{1}(\xi)\right]+r_{1} \phi_{1}(\xi)\left[1-b_{1}-\phi_{1}(\xi)\right]
$$

and

$$
\lim _{\xi \rightarrow-\infty} \phi_{1}(\xi) e^{-\lambda_{1}(c) \xi}=1
$$

Evidently, $\left(\underline{\phi}_{1}(\xi), \underline{\phi}_{2}(\xi)\right)$ is a lower solution to (2.1) (for the existence of $\underline{\phi}_{1}(\xi)$ and $\underline{\phi}_{1}(\xi) \leq$ $\min \left\{e^{\lambda_{1}(c) \xi}, 1-b_{1}\right\}$, we refer to Pan et al. [33]). By Lemma 2.2, we see that (2.1)-(2.2) has a monotone solution $\left(\phi_{1}(\xi), \phi_{2}(\xi)\right)$. Now, it suffices to prove Claim A.

If $\bar{\phi}_{1}(\xi)=1$ or $\bar{\phi}_{2}(\xi)=1$, the result is clear. If $\xi \leq 0$, then

$$
\bar{\phi}_{2}(\xi) \leq e^{\lambda_{1}(c) \xi} / b_{1}
$$

such that

$$
\begin{aligned}
& d_{1}\left[\int_{\mathbb{R}} J_{1}(\xi-y) \bar{\phi}_{1}(y) d y-\bar{\phi}_{1}(\xi)\right]-c \bar{\phi}_{1}^{\prime}(\xi)+r_{1} \bar{\phi}_{1}(\xi)\left[1-b_{1}-\bar{\phi}_{1}(\xi)+b_{1} \bar{\phi}_{2}(\xi)\right] \\
& \leq d_{1}\left[\int_{\mathbb{R}} J_{1}(\xi-y) e^{\lambda_{1}(c) y} d y-e^{\lambda_{1}(c) \xi}\right]-c \lambda_{1}(c) e^{\lambda_{1}(c) \xi} \\
& \quad+r_{1} e^{\lambda_{1}(c) \xi}\left[1-b_{1}-e^{\lambda_{1}(c) \xi}+b_{1} e^{\lambda_{1}(c) \xi} / b_{1}\right] \\
& =e^{\lambda_{1}(c) \xi} \Delta_{1}\left(\lambda_{1}(c), c\right)=0,
\end{aligned}
$$

which completes the proof on $\bar{\phi}_{1}(\xi)$ for $\xi \neq 0$.

We now consider $\bar{\phi}_{2}(\xi)<1$ with $\xi<0$. If $b_{1} b_{2} \geq 1$, then $b_{2} e^{\lambda_{1}(c) \xi} \geq e^{\lambda_{1}(c) \xi} / b_{1}$ such that

$$
b_{2} \bar{\phi}_{1}(\xi)-\bar{\phi}_{2}(\xi)=b_{2} e^{\lambda_{1}(c) \xi}-\frac{e^{\lambda_{1}(c) \xi}}{b_{1}} \geq 0
$$


and

$$
r_{2}\left[1-\bar{\phi}_{2}(\xi)\right]\left[b_{2} \bar{\phi}_{1}(\xi)-\bar{\phi}_{2}(\xi)\right] \leq r_{2}\left[b_{2} e^{\lambda_{1}(c) \xi}-\frac{e^{\lambda_{1}(c) \xi}}{b_{1}}\right]
$$

Therefore, (3.1) leads to

$$
\begin{aligned}
& d_{2}\left[\int_{\mathbb{R}} J_{2}(\xi-y) \bar{\phi}_{2}(y) d y-\bar{\phi}_{2}(\xi)\right]-c \bar{\phi}_{2}^{\prime}(\xi)+r_{2}\left[1-\bar{\phi}_{2}(\xi)\right]\left[b_{2} \bar{\phi}_{1}(\xi)-\bar{\phi}_{2}(\xi)\right] \\
& \quad \leq d_{2}\left[\int_{\mathbb{R}} J_{2}(\xi-y) \bar{\phi}_{2}(y) d y-\bar{\phi}_{2}(\xi)\right]-c \bar{\phi}_{2}^{\prime}(\xi)+r_{2}\left[b_{2} \bar{\phi}_{1}(\xi)-\bar{\phi}_{2}(\xi)\right] \\
& \quad \leq \frac{e^{\lambda_{1}(c) \xi}}{b_{1}}\left[d_{2}\left[\int_{\mathbb{R}} J_{2}(y) e^{\lambda_{1}(c) y} d y-1\right]-c \lambda_{1}(c)+r_{2}\left(b_{1} b_{2}-1\right)\right] \\
& \leq 0 .
\end{aligned}
$$

If $b_{1} b_{2}<1$, then $b_{2} \bar{\phi}_{1}(\xi)-\bar{\phi}_{2}(\xi) \leq 0$ and (3.2) imply that

$$
\begin{aligned}
& d_{2}\left[\int_{\mathbb{R}} J_{2}(\xi-y) \bar{\phi}_{2}(y) d y-\bar{\phi}_{2}(\xi)\right]-c \bar{\phi}_{2}^{\prime}(\xi)+r_{2}\left[1-\bar{\phi}_{2}(\xi)\right]\left[b_{2} \bar{\phi}_{1}(\xi)-\bar{\phi}_{2}(\xi)\right] \\
& \quad \leq d_{2}\left[\int_{\mathbb{R}} J_{2}(\xi-y) \bar{\phi}_{2}(y) d y-\bar{\phi}_{2}(\xi)\right]-c \bar{\phi}_{2}^{\prime}(\xi) \\
& \quad \leq \frac{e^{\lambda_{1}(c) \xi}}{b_{1}}\left[d_{2} \int_{\mathbb{R}} J_{2}(y) e^{\lambda_{1}(c) y} d y-d_{2}-c \lambda_{1}(c)\right] \\
& \leq 0
\end{aligned}
$$

Therefore, Claim A is true. The proof is complete.

Theorem 3.3 Assume that one of the following items holds.

(1) $b_{1} b_{2}>1$ and

$$
d_{2}\left[\int_{\mathbb{R}} J_{2}(y) e^{\lambda_{1}\left(c^{*}\right) y} d y-1\right]-c^{*} \lambda_{1}\left(c^{*}\right)+r_{2}\left(b_{1} b_{2}-1\right)<0
$$

(2) $b_{1} b_{2} \leq 1$ and

$$
d_{2}\left[\int_{\mathbb{R}} J_{2}(y) e^{\lambda_{1}\left(c^{*}\right) y} d y-1\right]-c^{*} \lambda_{1}\left(c^{*}\right)<0 .
$$

Then (2.1)-(2.2) has a monotone solution with $c=c^{*}$.

Proof If (3.3) or (3.4) holds, then there exists a decreasing sequence $\left\{c_{n}\right\}_{n=1}^{\infty}$ with $c_{n} \rightarrow c^{*}$, $n \rightarrow \infty$ such that for each $c_{n},(2.1)$-(2.2) has a positive monotone solution $\left(\phi_{1}^{n}, \phi_{2}^{n}\right)$. Note that a traveling wave solution is invariant in the sense of phase shift, so we can assume that

$$
\phi_{2}^{n}(0)=1 / 2
$$


for any $n$. By the Ascoli-Arzela lemma and a standard nested subsequence argument (see, e.g., Thieme and Zhao [47]), there exists a subsequence of $\left\{c_{n}\right\}_{n=1}^{\infty}$, which is still denoted by $\left\{c_{n}\right\}_{n=1}^{\infty}$ without confusion, such that $\left(\phi_{1}^{n}(\xi), \phi_{2}^{n}(\xi)\right)$ converges uniformly on every bounded interval, and hence pointwise on $\mathbb{R}$ to a continuous function $\left(\widehat{\phi}_{1}(\xi), \widehat{\phi}_{2}(\xi)\right)$. Moreover, for each $c_{n}$, we have

$$
\frac{1}{c_{n}} e^{-\frac{\beta}{c_{n}}(\xi-s)} \rightarrow \frac{1}{c^{n}} e^{-\frac{\beta}{c^{*}}(\xi-s)} \quad \text { for any } \xi \in \mathbb{R}, s \leq \xi,
$$

and the convergence in $s$ is uniform for $s \leq \xi$. Letting $n \rightarrow \infty$ and using the dominated convergence theorem in $\left(F_{1}, F_{2}\right)$, we know that $\left(\widehat{\phi}_{1}(\xi), \widehat{\phi}_{2}(\xi)\right)$ also satisfies $(2.1)$ with $c=c^{*}$. In addition, the following items are also clear.

(T1) $\widehat{\phi}_{2}(0)=1 / 2$ (by (3.5));

(T2) $\widehat{\phi}_{1}(\xi), \widehat{\phi}_{2}(\xi)$ are nondecreasing in $\xi$;

(T3) $0 \leq \widehat{\phi}_{1}(\xi), \widehat{\phi}_{2}(\xi) \leq 1, \xi \in \mathbb{R}$.

The items (T1) to (T3) further indicate that $\lim _{\xi \rightarrow \pm \infty} \widehat{\phi}_{i}(\xi)$ exists for $i=1,2$. Denote

$$
\lim _{\xi \rightarrow-\infty} \widehat{\phi}_{i}(\xi)=\widehat{\phi}_{i}^{-}, \quad \lim _{\xi \rightarrow \infty} \widehat{\phi}_{i}(\xi)=\widehat{\phi}_{i}^{+}, \quad i=1,2
$$

From (T1), it is clear that

$$
0 \leq \widehat{\phi}_{2}^{-} \leq \frac{1}{2} \leq \widehat{\phi}_{2}^{+} \leq 1 .
$$

If $\widehat{\phi}_{2}^{-} \in(0,1 / 2]$, then the dominated convergence theorem in $F_{2}$ implies that

$$
b_{2} \widehat{\phi}_{1}^{-}=\widehat{\phi}_{2}^{-} .
$$

Using the dominated convergence theorem in $F_{1}$ for $\xi \rightarrow-\infty$, we get the following possible conclusions:

(L1) $\widehat{\phi}_{1}^{-}=0$;

(L2) $1-b_{1}-\widehat{\phi}_{1}^{-}+b_{1} \widehat{\phi}_{2}^{-}=1-b_{1}-\widehat{\phi}_{1}^{-}+b_{1} b_{2} \widehat{\phi}_{1}^{-}=0$.

If (L1) is true, then the dominated theorem in $F_{2}$ tells us

$$
\widehat{\phi}_{2}^{-}\left[1-\widehat{\phi}_{2}^{-}\right]=0,
$$

which implies a contradiction. If (L2) is true, then $b_{1} b_{2}>b_{1}$ leads to

$$
0=1-b_{1}-\widehat{\phi}_{1}^{-}+b_{1} b_{2} \widehat{\phi}_{1}^{-}>1-b_{1}-\widehat{\phi}_{1}^{-}+b_{1} \widehat{\phi}_{1}^{-}=\left(1-\widehat{\phi}_{1}^{-}\right)\left(1-b_{1}\right)
$$

which is also a contradiction. What we have done implies that $\widehat{\phi}_{2}^{-}=0$. Using the dominated convergence theorem in $F_{2}$ again, we see that $b_{2} \widehat{\phi}_{1}^{-}=\widehat{\phi}_{2}^{-}=0$ and $\widehat{\phi}_{1}^{-}=0$.

If $\widehat{\phi}_{2}^{+} \in[1 / 2,1)$, then a discussion similar to that on $\widehat{\phi}_{2}^{-}$can be presented and we omit it here. Because $\widehat{\phi}_{2}^{+}=1$, then the dominated convergence in $F_{1}$ as $\xi \rightarrow+\infty$ indicates that $\widehat{\phi}_{1}^{+}=0$ or $\widehat{\phi}_{1}^{+}=1$. If $\widehat{\phi}_{1}^{+}=0$ is true, then $\phi_{1}(\xi) \equiv 0$ holds and

$$
\left\{\begin{array}{l}
c \phi_{2}^{\prime}(\xi)=d_{2}\left[\int_{\mathbb{R}} J_{2}(\xi-y) \phi_{2}(y) d y-\phi_{2}(\xi)\right]-r_{2} \phi_{2}(\xi)\left[1-\phi_{2}(\xi)\right] \\
\lim _{\xi \rightarrow-\infty} \phi_{2}(\xi)=0, \quad \lim _{\xi \rightarrow \infty} \phi_{2}(\xi)=1
\end{array}\right.
$$


has a monotone solution, which is impossible. Therefore, $\widehat{\phi}_{1}^{+}=1$ holds.

Thus, $\left(\widehat{\phi}_{1}(\xi), \widehat{\phi}_{2}(\xi)\right)$ is a positive monotone solution of (2.1)-(2.2) with $c=c_{*}$, the proof is complete.

\section{Nonexistence of traveling wave solutions}

In this section, we shall formulate the nonexistence of invasion traveling wave solutions of (1.2) by the theory of asymptotic spreading. Before this, we first present a comparison principle formulated by Jin and Zhao [34], Theorem 2.3.

Lemma 4.1 Assume that $\phi(x) \in C^{+}$. If $w(x, t) \geq 0, x \in \mathbb{R}, t>0$ is bounded such that

$$
\left\{\begin{array}{l}
\frac{\partial w(x, t)}{\partial t} \geq(\leq) d\left[\int_{\mathbb{R}} J(x-y) w(y, t) d y-w(x, t)\right]+r w(x, t)[1-w(x, t)] \\
w(x, 0) \geq(\leq) \phi(x), \quad x \in \mathbb{R}
\end{array}\right.
$$

then $w(x, t) \geq(\leq) u(x, t), x \in \mathbb{R}, t>0$.

We now give the main result of this section.

Theorem 4.2 If $c<c^{*}$, then (2.1)-(2.2) has no positive solutions.

Proof Define

$$
c_{2}=\inf _{\lambda>0}\left\{\frac{d_{1}\left[\int_{\mathbb{R}} J_{1}(y) e^{\lambda y} d y-1\right]+r_{1}\left(1-b_{1}\right)}{\lambda}\right\} .
$$

Then $c_{2}=c^{*}$ is evident.

If (2.1)-(2.2) has a positive solution $\left(\phi_{1}(\xi), \phi_{2}(\xi)\right)$ for some $c=\bar{c}<c^{*}$, then

$$
\phi_{2}(\xi)=\phi_{2}(x+\bar{c} t) \geq 0, \quad x \in \mathbb{R}, t>0, \xi \in \mathbb{R}
$$

implies that $\phi_{1}(\xi)$ also satisfies

$$
\bar{c} \phi_{1}^{\prime}(\xi) \geq d_{1}\left[\int_{\mathbb{R}} J_{1}(\xi-y) \phi_{1}(y) d y-\phi_{1}(\xi)\right]+r_{1} \phi_{1}(\xi)\left[1-b_{1}-\phi_{1}(\xi)\right]
$$

with the following asymptotic boundary condition:

$$
\lim _{\xi \rightarrow-\infty} \phi_{1}(\xi)=0, \quad \lim _{\xi \rightarrow \infty} \phi_{1}(\xi)=1
$$

Recalling the definition of traveling wave solutions, we see that $w(x, t)=\phi_{1}(x+\bar{c} t)$ also satisfies

$$
\frac{\partial w(x, t)}{\partial t} \geq d_{1}\left[\int_{\mathbb{R}} J_{1}(x-y) w(y, t) d y-w(x, t)\right]+r_{1} w(x, t)\left[1-b_{1}-w(x, t)\right]
$$

and

$$
0 \leq w(x, t) \leq 1, x \in \mathbb{R}, t \geq 0, \quad \lim _{x \rightarrow \infty} w(x, 0)=1
$$


Using Lemmas 2.4 and 4.1, we see that

$$
\lim _{t \rightarrow \infty} \inf _{2|x|=\left(\bar{c}+c^{\prime \prime}\right) t} w(x, t) \geq 1-b_{1}
$$

since $\bar{c}+c^{*}<2 c^{*}$.

However, the boundary condition (4.3) indicates that

$$
\xi=x+\bar{c} t \rightarrow-\infty \quad \text { with }-2 x=\left(\bar{c}+c^{*}\right) t, t \rightarrow \infty
$$

and

$$
\lim _{t \rightarrow \infty,-2 x=\left(\bar{c}+c^{*}\right) t} w(x, t)=0
$$

which implies a contradiction between (4.6) and (4.7). The proof is complete.

Remark 4.3 Under proper assumptions, we have obtained the threshold of the existence of positive solutions to (2.1)-(2.2).

\section{Competing interests}

The authors declare that they have no competing interests.

\section{Authors' contributions}

The main results in this article were derived by SP and GL. All authors read and approved the final manuscript.

\section{Author details}

'Department of Applied Mathematics, Lanzhou University of Technology, Lanzhou, Gansu 730050, People's Republic of China. ${ }^{2}$ School of Mathematics and Statistics, Lanzhou University, Lanzhou, Gansu 730000, People's Republic of China.

\section{Acknowledgements}

The authors express their thanks to the referees for their helpful comments and suggestions on the manuscript. This work was partially supported by the Development Program for Outstanding Young Teachers in Lanzhou University of Technology (1010ZCX019), NSF of China (11101094) and FRFCU (Izujbky-2011-k27).

\section{Received: 31 January 2012 Accepted: 8 October 2012 Published: 24 October 2012}

\section{References}

1. Ahmad, S, Lazer, AC, Tineo, A: Traveling waves for a system of equations. Nonlinear Anal. TMA 68, 3909-3912 (2008)

2. Fei, $\mathrm{N}$, Carr, J: Existence of travelling waves with their minimal speed for a diffusing Lotka-Volterra system. Nonlinear Anal., Real World Appl. 4, 503-524 (2003)

3. Gourley, SA, Ruan, S: Convergence and traveling fronts in functional differential equations with nonlocal terms: a competition model. SIAM J. Math. Anal. 35, 806-822 (2003)

4. Guo, JS, Liang, X: The minimal speed of traveling fronts for the Lotka-Volterra competition system. J. Dyn. Differ. Equ. 23, 353-363 (2011)

5. Hosono, Y: The minimal speed of traveling fronts for a diffusive Lotka-Volterra competition model. Bull. Math. Biol. 60, 435-448 (1998)

6. Huang, W: Problem on minimum wave speed for a Lotka-Volterra reaction-diffusion competition model. J. Dyn Differ. Equ. 22, 285-297 (2010)

7. Kan-on, Y, Fang, Q: Stability of monotone travelling waves for competition-diffusion equations. Jpn. J. Ind. Appl. Math. 13, 343-349 (1996)

8. Lewis, MA, Li, B, Weinberger, HF: Spreading speed and linear determinacy for two-species competition models. J. Math. Biol. 45, 219-233 (2002)

9. Li, WT, Lin, G, Ruan, S: Existence of traveling wave solutions in delayed reaction-diffusion systems with applications to diffusion-competition systems. Nonlinearity 19, 1253-1273 (2006)

10. Lin, G, Li, WT: Bistable wavefronts in a diffusive and competitive Lotka-Volterra type system with nonlocal delays. J. Differ. Equ. 244, 487-513 (2008)

11. Lin, G, Li, WT, Ma, M: Travelling wave solutions in delayed reaction diffusion systems with applications to multi-species models. Discrete Contin. Dyn. Syst., Ser. B 19, 393-414 (2010)

12. Lv, G, Wang, M: Traveling wave front in diffusive and competitive Lotka-Volterra system with delays. Nonlinear Anal., Real World Appl. 11, 1323-1329 (2010) 
13. Murray, LD: Mathematical Biology. Heidelberg, Springer (1989)

14. Tang, MM, Fife, P: Propagating fronts for competing species equations with diffusion. Arch. Ration. Mech. Anal. 73 69-77 (1980)

15. Wang, M, Lv, G: Entire solutions of a diffusive and competitive Lotka-Volterra type system with nonlocal delays. Nonlinearity 23, 1609-1630 (2010)

16. Yuan, Z, Zou, X: Co-invasion waves in a reaction diffusion model for competing pioneer and climax species. Nonlinear Anal., Real World Appl. 11, 232-245 (2010)

17. Shigesada, N, Kawasaki, K: Biological Invasions: Theory and Practice. Oxford University Press, Oxford (1997)

18. Guo, JS, Wu, CH: Traveling wave front for a two-component lattice dynamical system arising in competition models. J. Differ. Equ. 252, 4367-4391 (2012)

19. Yu, Z, Yuan, R: Travelling wave solutions in nonlocal reaction-diffusion systems with delays and applications. ANZIAM J. 51, 49-66 (2009)

20. van den Driessche, P: Spatial structure: patch models. In: Brauer, F, van den Driessche, P, Wu, J (eds.) Mathematical Epidemiology, pp. 179-189. Springer, Berlin (2008)

21. Bates, PW: On some nonlocal evolution equations arising in materials science. In: Brunner, H, Zhao, X, Zou, X (eds.) Nonlinear Dynamics and Evolution Equations. Fields Inst. Commun., vol. 48, pp. 13-52. Amer. Math. Soc., Providence (2006)

22. Bates, PW, Fife, PC, Ren, X, Wang, X: Traveling waves in a convolution model for phase transition. Arch. Ration. Mech. Anal. 138, 105-136 (1997)

23. Bates, PW, Han, J: The Neumann boundary problem for a nonlocal Cahn-Hilliard equation. J. Differ. Equ. 212, 235-277 (2005)

24. Bates, PW, Zhao, G: Existence, uniqueness and stability of the stationary solution to a nonlocal evolution equation arising in population dispersal. J. Math. Anal. Appl. 332, 428-440 (2007)

25. Carr, J, Chmaj, A: Uniqueness of travelling waves for nonlocal monostable equations. Proc. Am. Math. Soc. 132, 2433-2439 (2004)

26. Cortázar, C, Coville, J, Elgueta, M, Martínez, S: A nonlocal inhomogeneous dispersal process. J. Differ. Equ. 241, 332-358 (2007)

27. Ermentrout, B, Mcleod, J: Existence and uniqueness of travelling waves for a neural network. Proc. R. Soc. Edinb. A $123,461-478(1994)$

28. Fife, PC: Some nonclassical trends in parabolic and parabolic-like evolutions. In: Kirkilionis, M, Krömker, S, Rannacher, R, Tomi, F (eds.) Trends in Nonlinear Analysis, pp. 153-191. Springer, Berlin (2003)

29. Kao, CY, Lou, Y, Shen, W: Random dispersal vs. non-local dispersal. Discrete Contin. Dyn. Syst. 26, 551-596 (2010)

30. Shen, W, Zhang, A: Spreading speeds for monostable equations with nonlocal dispersal in space periodic habitats. J. Differ. Equ. 249, 747-795 (2010)

31. Yanagida, E, Zhang, L: Speeds of traveling waves in some integro-differential equations arising from neuronal networks. Jpn. J. Ind. Appl. Math. 27, 347-373 (2010)

32. Zhang, G: Global stability of wavefronts with minimal speeds for nonlocal dispersal equations with degenerate nonlinearity. Nonlinear Anal. 74, 6518-6529 (2011)

33. Pan, S, Li, WT, Lin, G: Travelling wave fronts in nonlocal delayed reaction-diffusion systems and applications. Z. Angew. Math. Phys. 60, 377-392 (2009)

34. Jin, Y, Zhao, XQ: Spatial dynamics of a periodic population model with dispersal. Nonlinearity $22,1167-1189$ (2009)

35. Coville, J, Dupaigne, L: Propagation speed of travelling fronts in nonlocal reaction-diffusion equation. Nonlinear Anal. TMA 60, 797-819 (2005)

36. Coville, J, Dupaigne, L: On a non-local equation arising in population dynamics. Proc. R. Soc. Edinb. A 137, 725-755 (2007)

37. Li, WT, Sun, Y, Wang, ZC: Entire solutions in the Fisher-KPP equation with nonlocal dispersal. Nonlinear Anal., Real World Appl. 11, 2302-2313 (2010)

38. LV, G: Asymptotic behavior of traveling fronts and entire solutions for a nonlocal monostable equation. Nonlinear Anal. TMA 72, 3659-3668 (2010)

39. Pan, S: Traveling wave fronts of delayed non-local diffusion systems without quasimonotonicity. J. Math. Anal. Appl. 346, 415-424 (2008)

40. Pan, S, Li, WT, Lin, G: Existence and stability of traveling wavefronts in a nonlocal diffusion equation with delay. Nonlinear Anal. TMA 72, 3150-3158 (2010)

41. Sun, Y, Li, WT, Wang, ZC: Traveling waves for a nonlocal anisotropic dispersal equation with monostable nonlinearity. Nonlinear Anal. TMA 74, 814-826 (2011)

42. Wu, S, Liu, S: Traveling waves for delayed non-local diffusion equations with crossing-monostability. Appl. Math. Comput. 217, 1435-1444 (2010)

43. $\mathrm{Xu}, \mathrm{Z}$, Weng, $\mathrm{P}$ : Traveling waves in a convolution model with infinite distributed delay and non-monotonicity. Nonlinear Anal., Real World Appl. 12,633-647 (2011)

44. Zhang, G, Li, WT, Wang, ZC: Spreading speeds and traveling waves for nonlocal dispersal equations with degenerate monostable nonlinearity. J. Differ. Equ. 252, 5096-5124 (2012)

45. Li, X, Lin, G: Traveling wavefronts in nonlocal dispersal and cooperative Lotka-Volterra system with delays. Appl. Math. Comput. 204, 738-744 (2008)

46. Zhang, G, Li, WT, Lin, G: Traveling waves in delayed predator-prey systems with nonlocal diffusion and stage structure. Math. Comput. Model. 49, 1021-1029 (2009)

47. Thieme, HR, Zhao, XQ: Asymptotic speeds of spread and traveling waves for integral equations and delayed reaction diffusion models. J. Differ. Equ. 195, 430-470 (2003)

doi:10.1186/1687-2770-2012-120

Cite this article as: Pan and Lin: Invasion traveling wave solutions of a competitive system with dispersal. Boundary Value Problems 2012 2012:120. 